\title{
Análisis económico de las vacunas del primer año de vida con componente pertussis en el contexto de la iniciativa mundial de erradicación de la poliomielitis en Argentina
}

\section{Vaccination in children under 1 year with pertussis component in the context of the global initiative for the eradication of poliomyelitis in Argentina: economic analysis}

\author{
Ángela Gentile', Norberto D. Giglio', Vanesa Castellano', Juan G. López² y Hugo Dibarboure³
}

'Hospital De Niños Ricardo Gutiérrez de Buenos Aires, Argentina.

${ }^{2}$ Sanofi Pasteur, México City, México.

${ }^{3}$ Sanofi Pasteur, Montevideo.

Conflictos de interés: A. Gentile, ND. Giglio y V. Castellano han realizado conferencias patrocinados por Sanofi Pasteur. H. Dibarboure y JG. López son empleados de Sanofi Pasteur.

Este estudio fue financiado por Sanofi Pasteur.

Recibido: 21 de octubre de 2020 / Aceptado: 3 de marzo de 2021

\section{Resumen}

Introducción: La Iniciativa Mundial de Erradicación de la Polio promueve la introducción de vacuna de polio inactivada (IPV) en sus programas, con la posterior retirada de Sabin (bOPV). Objetivo: Construir un modelo de económico que compare diferentes esquemas de vacunación para la prevención de polio y tosferina en el primer año de vida. Material y Métodos: Análisis de cuatro escenarios de vacunación del esquema primario para Argentina, en base a los precios de las vacunas, costos del programa y reactogenicidad de vacuna celular o acelular para Bordetella pertussis: - Escenario 1 (caso base): dos dosis de IPV, una dosis de bOPV y tres dosis de vacuna pentavalente (DTwP-HB-Hib); - Escenario 2: tres dosis IPV y de pentavalente; - Escenario 3: tres dosis de hexavalente (DTaPHepB-IPV-Hib); - Escenario 4: dos dosis de hexavalente más una dosis de pentavalente más IPV. Resultados: El costo incremental en base al escenario 1 fue de USD 3.716.671; 19.696 .668 y 14.383 .341 para los escenarios 2, 3 y 4, respectivamente. Para la reactogenicidad, la diferencia fue de USD -14.178.240 comparado el caso base con el escenario 3. Discusión: La inversión de incorporación de full IPV y costos asociados se modifica según tipo de vacuna y reactogenicidad asociada al componente $B$. pertussis.

Palabras clave: costos y análisis de costos, poliomielitis, vacuna contra la tosferina, esquemas de inmunización.

\section{Abstract}

Background: Global Polio Eradication Initiative promotes the introduction of inactivated polio vaccine (IPV) in its programs, with withdrawal of Sabin (bOPV). There is no an economic analysis of the investment related to the incorporation of IPV vaccines together with a whole cell Bordetella pertussis vaccine or combined with acellular hexavalent. Aim: An economic model that compares different vaccination schemes for the prevention of polio and pertussis in the first year of life was carried out. Methods: Four vaccination scenarios for the primary scheme based on Argentina demographic and costs data were developed: - Scenario 1 (base case): two doses of IPV, one dose of bOPV and three doses of pentavalent (DTwP-HepB-Hib) vaccine; - Scenario 2: three doses of IPV plus three doses of pentavalent; - Scenario 3: three doses of hexavalent; - Scenario 4: two doses of hexavalent plus one dose of pentavalent plus IPV. Results: The incremental cost based on scenario 1 was USD 3.716.671; 19.696.668 and 14.383.341 for scenarios 2, 3 and 4 respectively. In terms of reactogenicity savings was -14.178 .240 compared base case with scenario 3. Discussion: Full IPV introduction investment and costs associated were modified according to the type of vaccine and reactogenicity related with the $B$. pertussis component.

Keywords: costs and cost analysis, polio, pertussis vaccine, immunization schedules. 


\section{Material y Métodos}

Se utilizó como marco referencial los patrones demográficos y costos de Argentina.

En base al número de nacimientos de una cohorte hipotética de 728.035 niños $^{7}$ se realizó el análisis económico de cuatro escenarios de vacunación. A los fines de incluir el modelo de vacunación utilizado por algunos de los países de Latinoamérica, que incluyen todavía bOPV, se eligió escenario basal (escenario 1) el esquema primario de dos dosis de IPV y una dosis de bOPV junto con tres dosis de la vacuna pentavalente, (esquema iniciado para Argentina en mayo 2016 y finalizado en junio 2020). Este último, se comparó con tres escenarios: escenario 2, con tres dosis IPV más tres dosis de la vacuna pentavalente, esquema iniciado en junio 2020; el escenario 3, con tres dosis de la vacuna hexavalente; y el escenario 4, con dos dosis de la vacuna hexavalente más una dosis de la vacuna pentavalente e IPV. En todos los escenarios se consideró el mismo esquema para prematuros (8.078 prematuros con peso $<1.500 \mathrm{~g})$ que contempla tres dosis de vacuna hexavalente en el esquema inicial ${ }^{7}$.

Para la construcción del modelo se asumió similar eficacia y se analizaron las posibles diferencias, en relación con los costos asociados a reactogenicidad relacionados con el componente pertussis ${ }^{8,9}$ y los diferentes esquemas asociados a los programas de vacunación en términos de costos de almacenamiento, cadena de frío y del personal de salud destinado a la aplicación de las vacunas monodosis o combinadas.

En relación a los costos asociados a la reactogenicidad del componente de las vacunas acelulares (aP) y de células enteras (wP), la estimación de la incidencia se realizó en base a una revisión sistemática de la literatura, utilizando PUBMED y la base de datos del Sistema de Información de Ciencias de la Salud de América Latina y el Caribe (LILACS) (Ver Anexo). Los supuestos de probabilidad de uso de los recursos de salud relacionados con los eventos adversos se obtuvieron por consenso de expertos, compuesto por tres pediatras con experiencia en vacunología y dos pediatras generales. Los detalles de la estrategia de búsqueda se proporcionan en el material suplementario. En la Tabla 1 se describen las tasas de eventos adversos por dosis para vacunas de $B$. pertussis y el uso de recursos asociados. En la Tabla 2 se describen los costos médicos directos asociados cada evento adverso por dosis de vacuna y sus correspondientes asunciones.

El análisis costo de reactogenicidad se realizó desde una perspectiva social condición que agregó al modelo la pérdida de productividad de los cuidadores. Se asumió que $50 \%$ de los cuidadores estaban empleados ${ }^{10}$.

En relación al programa de inmunizaciones, para el 


\section{— -}

\begin{tabular}{|c|c|c|c|c|c|c|}
\hline \multirow[t]{2}{*}{ Evento adverso } & \multicolumn{3}{|c|}{ Incidencia (\%) } & \multicolumn{3}{|c|}{ Utilización de recursos (probabilidad) } \\
\hline & (wP) & (aP) & Referencia & Consultas & Hospitalización & Antipiréticos/analgésicos \\
\hline Fiebre & 26,20 & 2,58 & Korkmaz $2014^{20}$ & - & - & 1,0 \\
\hline Fiebre $38-39^{\circ} \mathrm{C}$ & 11,60 & 1,40 & & 0,8 & - & 1,0 \\
\hline Fiebre $>39^{\circ} \mathrm{C}$ & 4,50 & 0,02 & & 1,0 & - & 1,0 \\
\hline Somnolencia & 1,00 & 0,10 & & 0,1 & - & - \\
\hline Irritabilidad & 10,60 & 0,80 & & 0,3 & - & 0,8 \\
\hline Anorexia & 16,80 & 8,03 & Decker $1995^{21}$ & 0,3 & - & - \\
\hline Vómitos & 5,60 & 2,76 & & 0,1 & 0,1 & - \\
\hline Reacciones locales & 5,50 & 0,50 & Korkmaz $2014^{20}$ & 1,0 & 0,8 & 0,8 \\
\hline Convulsiones & 0,000150 & 0,000045 & Zielinski $2008^{22}$ & 1,5 & 0,7 & - \\
\hline Hipotonía-Hiporrespuesta EHH & 0,00025 & 0,00013 & Zielinski $2008^{22}$ & 0,9 & 0,7 & - \\
\hline
\end{tabular}

\begin{tabular}{|c|c|c|c|c|c|c|c|}
\hline \multirow[b]{2}{*}{$\begin{array}{l}\text { Costo por evento adverso } \\
\text { por dosis aplicada }\end{array}$} & \multicolumn{3}{|c|}{ Vacunas de células enteras (wP) } & \multicolumn{3}{|c|}{ Vacunas con componente acelular (aP) } & \multirow[t]{2}{*}{ Diferencia (USD) } \\
\hline & $\begin{array}{l}\text { Utilización de } \\
\text { servicios médicos* }\end{array}$ & Antipirético ${ }^{\dagger}$ & Total/dosis & $\begin{array}{l}\text { Utilización de } \\
\text { servicios médicos* }\end{array}$ & Antipirético $^{\dagger}$ & Total/dosis & \\
\hline Fiebre leve & - & 58.761 & 58.761 & - & 5.786 & 5.786 & -52.975 \\
\hline Fiebre moderada & 335.785 & 27.751 & 363.536 & 40.526 & 3.349 & 43.875 & -319.661 \\
\hline Fiebre severa & 549.036 & 10.765 & 559.802 & 2.440 & 48 & 2.488 & -557.314 \\
\hline Somnolencia & 3.618 & - & 3.618 & 362 & - & 362 & -3.256 \\
\hline Irritabilidad & 387.986 & 20.287 & 408.272 & 29.282 & 1.531 & 30.813 & -377.459 \\
\hline Anorexia & 182.366 & - & 182.366 & 87.167 & - & 87.167 & -95.199 \\
\hline Vómitos & 473.934 & - & 473.934 & 233.582 & - & 233.582 & -240.352 \\
\hline Reacciones locales & 199.011 & 10.526 & 209.537 & 18.092 & 957 & 19.049 & -190.488 \\
\hline Convulsiones & 313 & - & 313 & 94 & - & 94 & -219 \\
\hline EHH & 501 & - & 501 & 261 & - & 261 & -240 \\
\hline Total por dosis & & & 2.260 .640 & & & 423.477 & -1.837 .163 \\
\hline
\end{tabular}

cálculo de costos de almacenamiento y cadena de frío, se tuvieron en cuenta los volúmenes de caja o contenedores de las vacunas, de acuerdo al tipo de presentación de las vacunas en estudio. Se estimó un tiempo de permanecía en frigorífico central, además de gastos de trasporte a las diferentes regiones de Argentina ajustada por población. Se consideraron los precios de las vacunas, insumos y los costos del personal de salud para la administración de las mismas en base al salario promedio y tiempo insumido en la aplicación.

En la Tabla 3 se describen los costos unitarios del programa, salario promedio, gastos de bolsillo y sus correspondientes asunciones.

No se realizaron descuentos ya que el estudio tuvo un horizonte temporal de seis meses.

La evaluación de los costos de los diferentes escenarios 
Tabla 3. Costos unitarios del programa, salario promedio y gastos de bolsillo

\section{Costo USD}

\section{Tipo y costo por dosis vacunas e insumos}

Vacuna pentavalente (WP) (presentación en caja de 48 viales)

Vacuna IPV monodosis (jeringa prellenada)

Vacuna hexavalente (presentación en caja de 10 viales unidosis líquidos)

Jeringa (precio por unidad)
Referencia

1,08

5,3

20,6

0,03

$\mathrm{HNRG}^{26}$

Salario promedio vacunadora (hora)
Salunador

0,10

Pellissier $2000^{27}$

Costo según tiempo de aplicación de vacuna hexavalente (12,6 min)

1,26

1,50

Costo de la cadena de frío por dosis*

$\begin{array}{lc}\text { Vacuna pentavalente (wP) } & 0,003 \\ \text { Vacuna IPV monodosis (jeringa prellenada) } & 0,01 \\ \text { Vacuna OPV } & 0,0001 \\ \text { Vacuna pentavalente acelular (jeringa prellenada) } & 0,03 \\ \text { Vacuna hexavalente } & 0,002\end{array}$

Costo del trasporte por dosis

$\begin{array}{lr}\text { Vacuna pentavalente (wP) } & 0,001\end{array}$

$\begin{array}{lr}\text { Vacuna IPV monodosis (jeringa prellenada) } & 0,01\end{array}$

$\begin{array}{ll}\text { Vacuna OPV } & 0,0001\end{array}$

$\begin{array}{ll}\text { Vacuna pentavalente acelular (jeringa prellenada) } & 0,01\end{array}$

$\begin{array}{lr}\text { Vacuna hexavalente } & 0,001\end{array}$

Pérdida de productividad - Salario promedio ${ }^{\dagger}$

$\begin{array}{lc}\text { Mensual } & 1.227,6 \quad 6,80 \\ \text { Por hora } & 54,50\end{array}$

\section{Gastos de bolsillo}

$\begin{array}{lr}\text { Transporte a la clínica } & 6,00 \\ \text { Transporte a la emergencia } & 6,00 \\ \text { Costo diario por hospitalización (transporte, alimento, otros costos) } & 5,00\end{array}$

*Los costos totales de entrega se ajustaron a partir de las distancias estatales y la población de los recién nacidos para cada provincia del país. ${ }^{7 \dagger}$ Los costos de almacenamiento en frío por dosis se estimaron en base a una cotización de Frigorífico Onetto, Argentina, para 2.090 palets con un costo de almacenamiento anual de USD 903 por europalet (1,2 $\mathrm{m} \times 0,8 \mathrm{~m} \times 1,35 \mathrm{~m}$ ), con un costo total de USD 1888,18. Se supuso que los costos de transporte por palet eran de 80,35 USD por $\leq 400 \mathrm{~km}$; USD 1.000 para $>400-800 \mathrm{~km}$, y USD 125 para > $800 \mathrm{~km}$. "Supuestos: pérdida de productividad de tres horas al visitar una clínica sin cita previa; pérdida de productividad de cuatro horas visitando una clínica de emergencia, a excepción de convulsiones y EHH (seis horas más un día hábil por cada día de hospitalización*El costo de los recursos de atención médica se tomó del tarifario del gobierno de la Ciudad de Buenos Aires para hospitales públicos en 2018 28: U,D 5.04 para la visita ambulatoria, USD 16,96 para visita a emergencias, USD 65,68 para visita a la sala de emergencias con 6 h de observación y USD 158,14 para un día de hospitalización. † Para el uso de antipiréticos, el costo del paracetamol se tomó como USD 2,14 por $20 \mathrm{ml}{ }^{29}$, y el consumo para todos los tipos de fiebre se estimó teniendo en cuenta el peso del percentil 50 del lactante a los 2, 4, 6 meses a dosis estándar ( $\mathrm{mg} / \mathrm{kg}$ ) cada $8 \mathrm{~h}$ durante 1,2 días. Para la fiebre moderada o severa, el intervalo de dosis se estimó en $6 \mathrm{~h}$ durante 1,2 días ${ }^{30-33}$.

se sustentó en base a un modelo económico desarrollado por los investigadores de este estudio ${ }^{11}$.

Todos los parámetros analizados en el estudio se incluyeron en un modelo adaptable para la región en base al programa de Microsoft Excel.

Los costos se expresaron en dólares estadounidenses (USD) a una paridad cambiaria de 1 USD $=28$ pesos argentinos.

\section{Resultados}

Los costos totales para el caso base y los escenarios se resumen en la Tabla 4.

El costo incremental con base en el escenario 1 fue de USD 3.716.671; USD 19.696.668 y USD 14.383.341 para los escenarios 2, 3 y 4, respectivamente.

Para el caso específico de la Argentina, cuando 


\begin{tabular}{|c|c|c|c|c|}
\hline Escenarios & 1 (caso base) & 2 & 3 & 4 \\
\hline Vacunas y dosis & $\begin{array}{l}\text { IPV monovalente: } 2 \\
\text { dosis + bOPV: } 1 \text { dosis } \\
\text { pentavalente: } 3 \text { dosis }\end{array}$ & $\begin{array}{l}\text { IPV monovalente y } \\
\text { pentavalente: } 3 \text { dosis }\end{array}$ & Hexavalente: 3 dosis & $\begin{array}{l}\text { Hexavalente: } 2 \text { dosis } \\
\text { más IPV monovalente y } \\
\text { pentavalente: } 1 \text { dosis }\end{array}$ \\
\hline Costo de esquema por niño de 6 meses vacunado (USD) & 18,54 & 23,68 & 65,63 & 51,65 \\
\hline Costo de la vacuna programa (cobertura 100\%) (USD) & $13.348 .510,6$ & $17.051 .969,4$ & $47.252 .183,6$ & $37.185 .445,6$ \\
\hline Costo del Programa Prematuros (USD) (cobertura 100\%)* & $530.174,9$ & $530.174,9$ & $530.174,9$ & $530.174,9$ \\
\hline $\begin{array}{l}\text { Eventos adversos: costos directos, indirectos y pérdida de } \\
\text { productividad (USD) }\end{array}$ & 17.304.303,2 & $17.304 .303,2$ & $3.126 .062,9$ & 7.852 .143 \\
\hline Costo de la cadena de frío y transporte (USD) & $34.724,1$ & $47.936,3$ & $5.959,3$ & $33.290,5$ \\
\hline Costo total del escenario (USD) & $31.217 .712,8$ & $34.934 .383,8$ & $50.914 .380,7$ & 45.601 .054 \\
\hline
\end{tabular}

Figura 1. Distribución de costos para cada escenario de vacunación. *Los números en paréntesis indican el número de dosis.

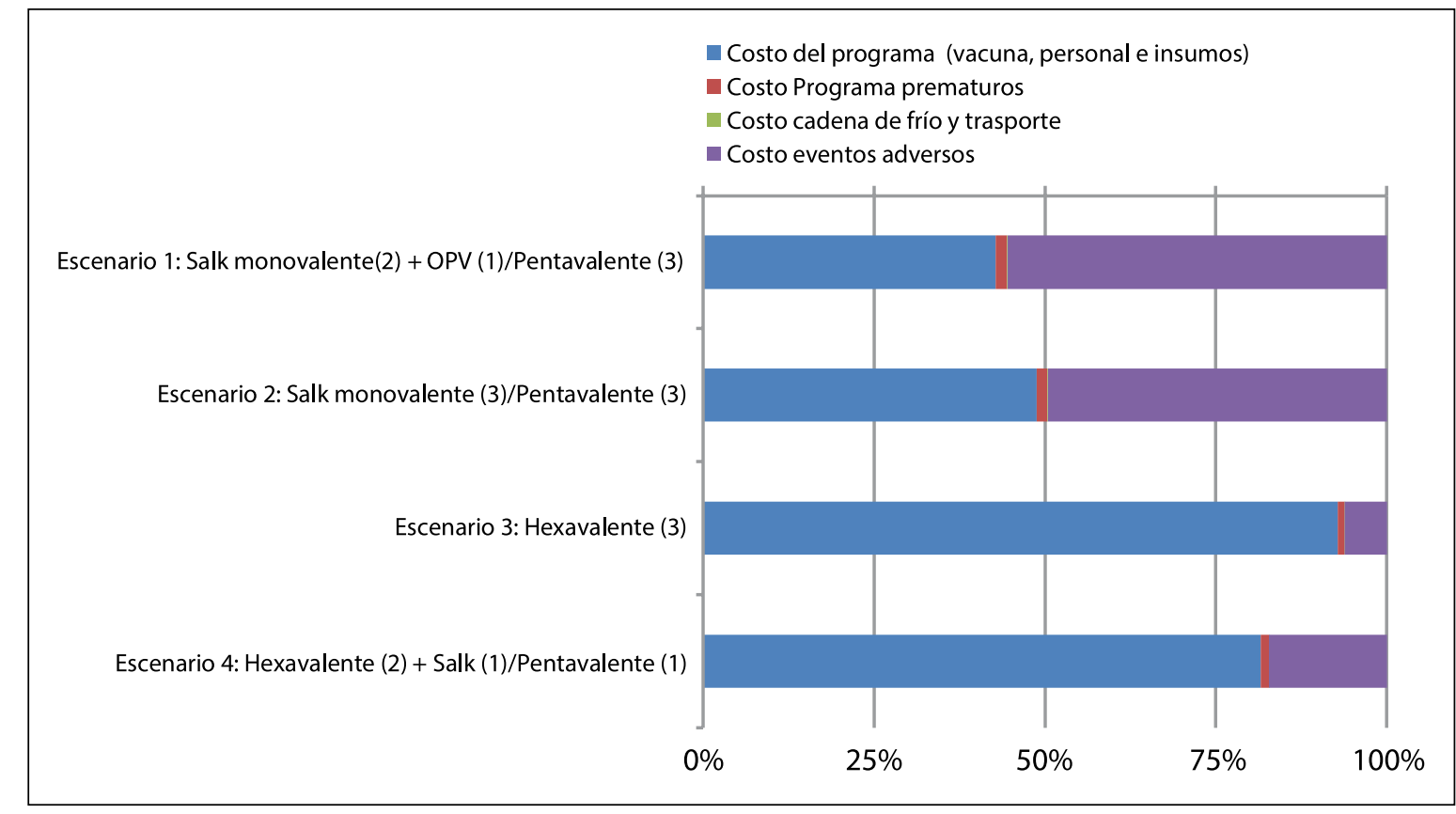

comparamos con el escenario actual modificado en junio 2020 (escenario 2), los costos incrementales de posibles modificaciones de esquema fueron de USD 15.979.997 y de USD 10.666.670 para el escenario 3 y 4 respectivamente. Cuando se compara la reactogenicidad del esquema completo con wP (escenarios 1 y 2) con un esquema completo con aP (escenario 3) la diferencia de costos significó un gasto evitado en reactogenicidad para las vacunas acelulares de USD -14.178.240. En el mismo sentido, los gastos de cadena de frío y transporte mostraron un ahorro para el escenario 3 comparado con el escenario 1 de USD -28.765.
Finalmente, y en relación con el precio de las vacunas, el escenario 3 resulto el más costoso cuando se comparó con los escenarios restantes. En la Figura 1 se describen la representatividad de los costos analizados para cada escenario.

\section{Discusión}

Los decisores vinculados a los programas nacionales de vacunación, en estos momentos enfrentan desafíos para incorporar nuevos esquemas de inmunización ante la 
necesidad de cumplir con los lineamientos de la Estrategia Global para la Erradicación de la poliomielitis.

En este sentido, nuestro estudio permitió dimensionar en término de evidencia cuál fue el costo que implicó en un escenario con datos argentinos, finalizar el uso de bOPV, introducir un esquema completo con IPV y cómo impactarían los costos en el programa si potencialmente se utilizara una vacuna hexavalente acelular en el esquema inicial.

En el modelo de reemplazo de una dosis de bOPV por IPV administrada con vacuna pentavalente en el esquema inicial, se observó un costo incremental de USD 3,7 millones que incluye costos de la vacuna, cadena de frío /distribución y no ha modificado el gasto de la reactogenicidad vinculada al componente wP.

El costo por el manejo de los eventos adversos representó aproximadamente el $50 \%$ de los costos totales para el programa finalizado en mayo 2020 y el actual (escenario 2) (Figura. 1). En el mismo sentido, nuestros resultados revelan que, si se cambia la vacuna pentavalente de células completas por hexavalente acelular, en relación a los costos de los eventos adversos relacionados, reportamos un ahorro para las vacunas hexavalentes de USD 14,1 millones.

Por otro lado, también hemos podido observar que se reducen los costos del programa en lo que tiene que ver con almacenamiento y distribución de vacunas en cadena de frío, siendo el ahorro de tres dosis de hexavalente de USD 28.764 comparado con el escenario base y de $\sim$ USD 42.000 en comparación con el esquema actual. La problemática de los costos de distribución de vacunas en cadena de frío ya se ha identificado previamente ${ }^{12,13}$. Nosotros hemos encontrado que el esquema actual, con la incorporación de IPV incrementa el costo del programa en cadena de frío, distribución y recursos en salud (USD 13.212) al igual que lo describe un estudio desarrollado en Brasil por Sartori y cols. ${ }^{14}$.

Nuestro estudio presenta algunas limitaciones. En relación con la efectividad de las vacunas con componente wP y aP no hemos considerado posibles escenarios de diferencias para justificar la superioridad de una vacuna sobre la otra ${ }^{15}$. De todas maneras, el programa de control de enfermedad por $B$. pertussis en nuestro país no sólo considera a los lactantes bajo un año de edad, sino que contempla una estrategia integral que incluye refuerzos periódicos en el segundo año de vida, al ingreso escolar, vacunación en adolescentes y vacunación sistemáticas en mujeres embarazadas 5 .

En términos monetarios, la inestabilidad en la economía de Argentina ha alterado la tasa de convertibilidad $\mathrm{y}$, en consecuencia, los valores pronosticados en este estudio, y si bien no se ha considerado al sector privado, en nuestro país representa una minoría ${ }^{16}$. No obstante, los métodos utilizados en este análisis son sólidos y permiten una valoración útil y relevante del impacto de la incorporación de diferentes esquemas tomando como referencia el Programa Nacional de Inmunización en Argentina.

Frente al desafío en el que se encuentra Latinoamérica, este estudio permitirá sentar las bases de un modelo epidemiológico de análisis para futuros cambios de estrategias y por ende del calendario de los diversos países de la región.

Futuros estudios también serán necesarios para evaluar en cada país aspectos demográficos, costos sanitarios y sociales, además de profundizar el impacto de vacunas combinadas sobre las coberturas. Su uso podría aumentar la confianza en las vacunas por la disminución en la reactogenicidad y el número de inyecciones dentro de los beneficios que ofrecen las vacunas combinadas ${ }^{17}$. En el mismo sentido, el reemplazo de OPV por IPV justifica también la inversión debido al costo que implica la parálisis fláccida asociada OPV y sus secuelas a largo plazo $^{18}$. La reducción de estas entidades fortalecerá la confianza de la comunidad en las vacunas y por ende en los programas de vacunación ${ }^{19}$.

\section{Conclusiones}

La inversión actual hacia un esquema completo de IPV generó para la Argentina un aumento de los costos del programa. El uso de una vacuna hexavalente reduce los costos asociados del programa y la reactogenicidad, que compensa parcialmente el costo de la vacuna. La inversión tendría beneficios adicionales a largo plazo vinculados a la eliminación de parálisis fláccidas asociadas a los virus polio vacunales.

Agradecimientos A Pablo Bianculli por su apoyo y revisión de este manuscrito. 


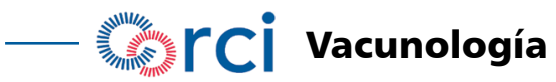

\section{Anexo. Motores de búsqueda utilizados para la revisión de la literatura}

Languages: English, Spanish, Portuguese and French.

MESH terms in PubMed

Acellular vaccines:

"Diphtheria-Tetanus-acellular Pertussis Vaccines"[MESH] OR Pertussis Vaccines, Diphtheria-Tetanus-acellular*[TIAB] OR Vaccines, Diphtheria-Tetanus-acellular Pertussis* $[T I A B]$ OR DTaP Vaccines * $[T I A B]$ OR Vaccines, DTaP*[TIAB] OR Vaccine, DTaP*[TIAB] OR Tripedia*[TIAB] OR Infanrix*[TIAB] OR Hexaxim*[TIAB] AND Adverse Effects AND "children"[TIAB]

Filters: From 2007/07/18 to 2017/07/18, Humans, Child: birth-18 years

Whole-cell vaccines:

Search: "Diphtheria-Tetanus-Pertussis Vaccine"[MESH] OR Diphtheria Tetanus Pertussis Vaccine*[TIAB] OR Vaccine, Diphtheria-Tetanus-Pertussis*[TIAB] OR DTwP Vaccine*[TIAB] OR Vaccine, DTwP*[TIAB] OR DPT Vaccine*[TIAB] OR Vaccine, DPT*[TIAB] OR DTP Vaccine*[TIAB] OR Vaccine, DTP*[TIAB] OR Di-Te-Per Vaccine*[TIAB] OR Di Te Per Vaccine*[TIAB] OR Vaccine, Di-Te-Per*[TIAB] OR Diphtheria-Pertussis-Tetanus Vaccine*[TIAB] OR Diphtheria Pertussis Tetanus Vaccine*[TIAB] OR Vaccine, Diphtheria-Pertussis-Tetanus*[TIAB] OR Heberpenta*[TIAB] OR Pentavalent*[TIAB] AND Adverse Effects AND "children"[TIAB]

Filters: From 2007/07/18 to 2017/07/18, Humans, Child: birth-18 years

Search terms in Lilacs

(instance:"regional") AND ( mh:("Vacuna contra la Tos Ferina/AE" OR "Vacuna contra Difteria, Tétanos y Tos Ferina/AE" OR "Vacunas contra Difteria, Tétanos y Tos Ferina Acelular/AE") AND db:("LILACS") AND limit:(“humans" OR "infant") AND year_cluster:("2007" OR "2008" OR "2009" OR "2010" OR "2011" OR "2012" OR "2013" OR "2014" OR "2015" OR "2016" OR "2017"))

\section{Referencias bibliográficas}

1.- Polio Global Eradication Initiative. Polio Eradication and Endgame Strategic Plan 2013-2018. 2013 Disponible:_http://www. polioeradication.org/resourcelibrary/ strategyandwork.aspx (acceso febrero de 2018).

2.- Organización Mundial de la Salud. Estrategia posterior a la Certificación de la Erradicación de la Poliomielitis. 2018. Disponible $\mathrm{http}: / /$ polioeradicationorg/wp-content/ uploads/2018/04/polio-post-certificationstrategy-20180424-sp.pdf (acceso enero de 2018).

3.- Dirección de Control de Enfermedades Inmunoprevenibles. Lineamientos técnicos. Nuevo esquema de Vacunación Antipoliomielítica. Año 2020. Disponible en: http://www.msal.gob.ar/images/stories/ bes/graficos/0000001964cnt-vacunacionantipoliomielitica_lineamientos 2020.pdf (acceso julio de 2020).

4.- Ministerio de Salud Argentina. Calendario Nacional de Vacunación 2017. Available at: http://www.msal.gob.ar/indexphp/programasy-planes/184-calendario-nacional-devacunacion-2016 (acceso enero de 2018).

5.- Ministerio de Salud. Gobierno de Chile. Puesta al día Calendario de Vacunación. Departamento de Inmunizaciones. Disponible en: https:// diprece.minsal.cl/wp-content/uploads/2019/07/ PNI-2019.pdf (acceso julio de 2020).
6.- WHO vaccine-preventable diseases: monitoring system. 2020 global summary Disponible at: https://apps.who.int/immunization_monitoring/ globalsummary/schedules (acceso agosto de 2020).

7.- Direcciòn de Estadísticas e Información en Salud. Estadísticas Vitales. Información básica. Año 2016. 2017. Disponible: http://www. deis.msal.gov.ar/wp-content/uploads/2018/06/ Serie5Nro60.pdf. (acceso julio de 2020).

8.- Zhang L, Prietsch SOM, Axelsson I, Halperin SA. Acellular vaccines for preventing whooping cough in children. Cochrane Database of Systematic Reviews 2014, Issue 9. Art. No.: CD001478. doi: 10.1002/14651858. CD001478.pub6.

9.- Global Advisory Committee on Vaccine Safety (GACVS). Information sheet observed rate of vaccine reactions diphtheria, pertussis, tetanus vaccines. WHO. Disponible en: https://www. who.int/vaccine_safety/initiative/tools/DTP vaccine_rates_information_sheet.pdf (acceso julio de 2020).

10.- Ministerio de Salud y Desarrollo Social de Argentina. Reporte del Trabajo Registrado 2018. 2019. Disponible: http://www.trabajo. gob.ar/estadisticas/Reporte Laboral Mayo_2018.pdf. (acceso julio de 2020)

11.- Gentile A, Giglio N, Castellano V, López J G, Dibarboure H. Análisis económico de vacuna hexavalente en Argentina en el marco de la Iniciativa Global para la Erradicación de Polio.
Modalidad poster $\left(\mathrm{N}^{\circ} 142\right)$. Congreso SLIPE (Sociedad Latinoamericana de Infectología Pediátrica). Agosto de 2019. Cartagena de India, Colombia. https://slipe.org/web/ publicaciones/ (acceso diciembre de 2019).

12.- Scott N, Palmer A, Morgan C, Lesi O, Spearman C W, Sonderup M, et al. Costeffectiveness of the controlled temperature chain for the hepatitis B virus birth dose vaccine in various global settings: a modelling study. Lancet Glob Health 2018; 6: e659-e67. doi: 10.1016/S2214-109X(18)30219-5.

13.- Seaman C P, Morgan C, Howell J, et al. Use of controlled temperature chain and compact prefilled auto-disable devices to reach 2030 hepatitis B birth dose vaccination targets in LMICs: a modelling and costoptimization study. Lancet Glob Health. 2020; 8 (7): e931-e941. doi:10.1016/S2214-109X (20)30231-X

14.- Sartori A M, Vicentine M P, Gryninger L C, Soárez P C, Novaes H M. Polio inactivated vaccine costs into routine childhood immunization in Brazil. Rev Saude Publica. 2015; 49: 8. doi:10.1590/s00348910.2015049005492 .

15.- WHO. Pertussis vaccines: WHO position paper-August 2015. No. 35, 2015, 90, 433-60. Disponible en: https://www.who.int/wer/2015/ wer9035.pdf?ua=1 (acceso julio de 2020).

16.- Rubinstein A, Zerbino M C, Cejas C, López A. Making universal health care effective in 
Argentina: A blueprint for reform. Health Syst Reform. 2018; 4: 203-13. https://doi.org/10.108 $0 / 23288604.2018 .1477537$.

17.- Khaled $\mathrm{M}$ et al. The value of childhood combination vaccines: From beliefs to evidence. Human Vaccines Immunother. 2015 Sept; 11 (9): 2132-41. doi: 10.1080/21645515.2015.1044180.

18.- Alvis N, De la Hoz F, Narvaez J. Vacuna inyectable contra la poliomielitis en Colombia. Impacto económico de la introducción de la vacuna inactivada inyectable contra la poliomielitis en Colombia. Rev Panam Salud Pública. 2010; 27 (5): 352-9. https://www. scielosp.org/article/rpsp/2010.v27n5/352-359/

19.- Chard A N, Datta S D, Tallis G, Burns C C, Wassilak S G F, Vertefeuille J F, et al. Progress toward polio eradication-worldwide, January 2018-March 2020. MMWR Morb Mortal Wkly Rep. 2020; 69: 784-9. doi: http://dx.doi. org/10.15585/mmwr.mm6925a4.

20.- Korkmaz H A, Aydin A, Unal B. Comparison of acellular pertussis-tetanus-diphtheria vaccines and whole-cell pertussis-tetanusdiphtheria vaccines in infancy. Paediatr Int Child Health. 2014; 34: 198-202. doi: 10.1179/2046905513Y.0000000110.

21.- Decker M D, Edwards K M, Steinhoff M C, Rennels M B, Pichichero M E, Englund J A, et al. Comparison of 13 acellular pertussis vaccines: adverse reactions. Pediatrics. 1995; 96: 557-66. PMID: 7659476.

22.- Zielinski A, Rosinska M. Comparison of adverse effects following immunization with vaccine containing whole-cell vs. acellular pertussis components. Przegl Epidemiol. 2008; 62: 589-96. PMID: 19108523.

23.- Castellano V, Pacchiotti A, Umido V, Lamy V, Nolte M F, Gentile A. Episodios de Hipotonía Hiporrespuesta (EHH) postvacunación: reporte de una serie de casos. Rev Hosp Niños (B Aires). 2018; 60: 29-33. http://revistapediatria. com.ar/wp-content/uploads/2018/05/268-06Episodios-de-hipotonia.pdf.

24.- Pan American Health Organization. Expanded program of immunization vaccine prices for year 2018. https://www.google.de/search?q=Pa $\mathrm{n}+$ American+Health+Organization. + Expanded + program + of + immunization + vaccine + prices $+f$ or+year+2018.\& trackid=sp-006 (acceso agosto de 2018).

25.- Expanded Program of Immunization. OPSWHO. Prices for years 2018-2019. https:// www.paho.org/hq/index.php?option=com docman\&task $=$ doc_download\&gid $=43416$ \& Ite mid=270\&lang=pt (acceso julio de 2020)

26.- Departamento de Recursos Humanos Hospital de Niños Ricardo Gutiérrez. Año 2018. https:// www.buenosaires.gob.ar/hospitalgutierrez (acceso agosto de 2018).

27.- Pellissier J M, Coplan P M, Jackson L A, May J E. The effect of additional shots on the vaccine administration process: results of a time-motion study in 2 settings. Am J Manag Care. 2000; 6: 1038-44. PMID: 11184065.

28.- Giglio N D, Caruso M, Castellano V E, Choque L, Sandoval S, Micone P, et al. Hospitalization costs associated with diarrhea among children during the period of rotavirus circulation in the Northwest region of Argentina. Arch Argent Pediatr. 2017; 115: 527-32. doi: 10.5546/ aap.2017.eng.527.

29.- Gentile A, Salgueiro A L, Garcia Bournissen F, Romanin V, Bulgheroni S, Gaiano A, et al. Cost of Bordetella pertussis illness in tertiary hospitals in Argentina. Arch Argent Pediatr. 2013; 111: 295-302. https://www.sap. org.ar/docs/publicaciones/archivosarg/2013/ v111n4a05e.pdf.

30.- Ministerio de Salud. Gobierno de la Ciudad de Buenos Aires. Comisión de actualización permanente del nomenclador de prestaciones de salud. 2018. https://documentosboletinoficial. buenosaires.gob.ar/publico/PE-RES-MSGCMSGC-404-18-ANX.pdf. (acceso agosto de 2018).

31.- Administración Nacional de Medicamentos, Alimentos y Tecnología Médica (ANMAT). Vademécum. Precio de los medicamentos. Disponible en: https://www.argentina.gob.ar/ anmat (acceso enero de 2018).

32.- Comité Nacional de Crecimiento y Desarrollo. Guías para la evaluación del crecimiento físico. Sociedad Argentina de Pediatría. República Argentina 2013. 2013. Available at: https:// www.sap.org.ar/docs/publicaciones/libro verde_sap_2013.pdf (acceso julio de 2020).

33.- Rousseau M, Sberna N, Calle G, Rodríguez M. Formulario Farmacoterapéutico 2016. Hospital Garrahan. 2016. https://farmacia.garrahan.gov. ar/Vademecum/Busqueda (acceso enero de 2019). 